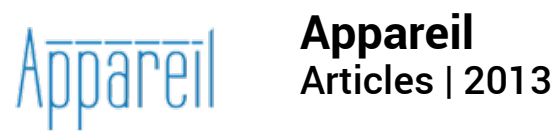

\title{
Le 6 juin 1944. La ligne d'horizon
}

\section{Jean-Louis Déotte}

\section{OpenEdition}

\section{Journals}

Édition électronique

URL : http://journals.openedition.org/appareil/1536

DOI : 10.4000/appareil.1536

ISSN : 2101-0714

Éditeur

MSH Paris Nord

\section{Référence électronique}

Jean-Louis Déotte, «Le 6 juin 1944. La ligne d'horizon », Appareil [En ligne], Articles, mis en ligne le 22

février 2013, consulté le 30 juillet 2020. URL : http://journals.openedition.org/appareil/1536 ; DOI : https://doi.org/10.4000/appareil.1536

Ce document a été généré automatiquement le 30 juillet 2020.

\section{(c) (i) (9)}

Appareil est mis à disposition selon les termes de la Licence Creative Commons Attribution - Pas d'Utilisation Commerciale - Pas de Modification 4.0 International. 


\title{
Le 6 juin 1944. La ligne d'horizon
}

\author{
Jean-Louis Déotte
}

\section{NOTE DE L'AUTEUR}

Ce texte reprend un travail sur le tourisme de guerre, publié par le FRAC de BasseNormandie (Caen) lors de l'exposition de Diller/Scofidio : « Back to the Front : Tourisms of war » (Visite aux armées : tourismes de guerre). Caen, 1994. Je remercie S. Zavatta, directrice du FRAC.

1 Si l'on reprend les traités de perspective et les traités de peinture, dont le premier pour l'époque moderne fut le Della Pictura d'Alberti, il est évident que suivant l'ordre méthodologique et constructif, le tracé de l'horizon précède le positionnement du point de fuite. Point de fuite appelé aussi chez Viator point du sujet. L'on sait aussi que si les artisans grecs travaillant pour les Romains élaborèrent les premiers un plan de tableau immatériel, transparent, ils ne conçurent pas l'espace ainsi représenté systématiquement. Ils ne cherchaient pas à faire se conjoindre les lignes constructives (ou lignes de fuite) en un seul point valant pour l'infini de ces lignes (point de fuite: Panofsky). Et ainsi ils n'eurent pas besoin d'une ligne constructive horizontale, mais plutôt d'une verticale, d'un axe, servant ainsi d'épine dorsale à ce que les spécialistes appellent une structure en arête de poisson.

2 Ce qui implique philosophiquement qu'entre les Anciens et les Modernes, le partage est entre le privilège de la verticalité et celui de l'horizontalité. Et que les Anciens ne purent déduire l'existence d'un sujet comme site nouveau de la vérité (Hegel à propos de Descartes) à partir de ce point géométrique valant pour toutes les droites du plan de base, idéalement prolongées à l'infini. L'Antiquité n'aurait pas fait de projet n'ayant pas eu d'horizon, comme elle ignora le sujet de la philosophie et l'infini.

Le sujet moderne de la peinture de représentation a donc immédiatement eu l'horizon pour l'action. Puisque dans la peinture du Quattrocento (si l'on suit Alberti), immédiatement après avoir fictivement ouvert et circonscrit la vitre du tableau (ce cadre carré qui découpe la vue à venir), la ligne d'horizon doit être tracée. Et cela, 
parallèlement à la ligne de base, deux tiers au-dessus. Or, c'est sur cette ligne que sera choisi, arbitrairement, le point du sujet. Le sujet, en son point, au fond du tableau, ne tient donc qu'à un fil, sur lequel il peut d'ailleurs bouger. Ce sujet, encore tout à fait abstrait, surgit de l'intérieur du tableau. Cette peinture de représentation privilégiera toujours l'inclusion et l'immanence contre l'exclusion et la transcendance médiévales.

Le point du sujet une fois choisi, les lignes de fuite peuvent être dessinées. Et en quelque sorte par projection symétrique de ce point du sujet hors du tableau, la position idéale du spectateur pourra être déterminée. Le point du sujet, intérieur, précède donc l'établissement du point de vue extérieur, ou de ce qu'on appelle communément le sujet (le spectateur). La conséquence centrale de cette construction est que le sujet est toujours déjà-là comme point, à l'intérieur du dessin ou du tableau, précédant donc toute vue effective. Ce n'est donc pas la vision de ce tableau par un sujet extérieur préexistant qui le subjectiviserait. Car celui qui voit ne subjectivise la vue qu'en découvrant qu'il avait déjà sa place - comme sujet - à l'intérieur du tableau ou de la scène de théâtre à l'italienne (Théâtre olympique à Vicenza). Bref, celui qui voit ne devient sujet, au sens des Méditations cartésiennes comme de toute la tradition philosophique moderne, que comme effet d'un appareil qui fit époque : la perspective. Appareil de projection dont Brunelleschi fit connaitre quelques uns des aspects spéculatifs les plus remarquables. Voir, comme spectateur, le point de fuite, c'est se retrouver dans le tableau déjà en position de maîtrise, a priori.

5 Ce qui nous importe ici, c'est la série des décisions méthodologiques et ontologiques: décider que dorénavant le monde peut être donné dans une vue, que ce n'est plus la face visible du texte sacré, qu'un quadrilatère dessiné sur un mur peut avoir la quasisubstance d'une vitre à travers laquelle on apercevrait ce monde, inscrire sur cette vitre les traces de cette chose qui est là-bas, donc l'objectiver, tracer la ligne d'horizon sur laquelle on placera le point du sujet, " projeter vers l'extérieur » le point de vue obtenu symétriquement, obtenir à partir de là les points de distance pour achever la rationalisation de l'espace ainsi représenté.

6 Notre hypothèse consiste donc à penser que cette série méthodologique, de pratique professionnelle, a une valeur ontologique. Celle d'une déduction des conditions de possibilité de la subjectivité, non seulement philosophique, mais aussi anthropologique et donc aussi bien politique, juridique, psychologique, économique, etc. Donc que les peintres et les architectes ont eu en ce domaine une préséance absolue sur les physiciens, les géomètres, les philosophes.

7 Si le sujet a toujours, depuis lors, un horizon (pour sa volonté par exemple : il ne pourrait faire de projets sans l'horizon du projet, etc.), c'est que l'horizon est sa condition de possibilité. Et comme l'indique le grec horizein, sa borne. Cette borne est la nouvelle définition de notre finitude. Elle précède ce qu'elle borne. À savoir l'expérience du champ qui lui est ainsi ouvert de ce point de vue-là. L'expérience du sujet moderne tiendra donc à être exclusivement horizontale, rabattant la transcendance du divin sur l'immanence de ce qui se projette comme traces pour un sujet sur un écran transparent. Donc, ce qu'on appelle l'humanisme est une forme première de l'immanentisme.

8 Comme le montre la série des perspecteurs portables, ces dispositifs purement techniques, du portillon de Dürer à celui de P. Greenaway (Meurtre dans un jardin anglais, ou plutôt selon le titre original Le Contrat du dessinateur), le dispositif de projection inclut toujours le viseur télémétrique. La cible n'étant rien d'autre que la chose là-bas 
venant se projeter comme objet sur la vitre orthonormée du plan de projection. En y laissant des points, qui sont autant de traces que le dessinateur doit relier. Il n'y a pas de différence de substance entre l'œil derrière le viseur et la cible qui est là-bas en face, mais une réversibilité toujours possible. La modernité confond allègrement le sujet et l'objet : ne dit-on pas d'un roman qu'il a un sujet alors qu'on vise par là son objet, ce dont il est question? Ainsi dans le combat moderne, le viseur et le visé appartiennent donc nécessairement à la sphère de la subjectivité. L'objet visé par ego est un alter ego, la relation entre les deux est immédiatement inter-subjective. L'intersubjectivité n'est pas une donnée immédiate, mais une conséquence de l'appareil projectif. Elle n'est donc pas à démontrer philosophiquement à partir de cet acquis indubitable que serait ego cogito (contrairement à Descartes et à Husserl).

9 Les ennemis modernes, avant de s'affronter, se visent et se transforment ainsi en sujets-objets les uns pour les autres. Ce n'est donc pas la supposée commune appartenance à l'humanité qui rend possible la fraternisation, comme à Verdun dès 1916. Inutile ici de supposer un sentiment de l'humain toujours déjà présent. Ce sentiment est la conséquence d'une certaine détermination époquale de la surface d'inscription, ici sous les traits de l'appareil perspectif. La figure de l'ennemi est donc sous la dépendance du dispositif technique et mental grâce auquel il est conçu et perçu, par ce que Cassirer appelait une forme symbolique.

10 A contrario, dans leurs guerres interminables, les communautés amérindiennes décrites par Clastres, considéraient l'autre, non comme humain (attribut qu'elles se réservaient chacune dans son unicité), mais comme existant hors humanité, fantôme ou animal (œuf de poux selon la traduction de Levi-Strauss). N'était semblable (donc humain) que ce qui portait sur soi les mêmes traits d'écriture corporelle, les mêmes scarifications, les mêmes découpes. Un autre vocabulaire de scarifications, d'autres tracés sur les corps, ne rendaient pas semblables (la formule: «tous sont humains parce que tous sont écrits, même si les signes diffèrent ", n'aurait pas été de mise, étant plutôt chrétienne). L'identification des tracés d'une autre tribu ne la faisait pas entrer dans le cercle d'une humanité restreinte. Ce type d'écriture, car il s'agit bien d'une écriture, d'un système différentiel de marques et d'entailles, d'incisions comme d'excisions, rabattait l'universel du lisible et donc du sens sur la singularité communautaire. L'écriture ne donnait pas accès à l'universel du sens, étant totalement territorialisée, idiomatique, absolument non-vocalisable, donc illisible au sens strict, tant la marque ou la lettre encageait singulièrement le sens comme chez Michaux. C'est par illusion d'optique que nous croyons pouvoir lire ces traits, en leur donnant un statut de "symboles" graphiques, ouverts au commentaire, indéfiniment. Leurs graphèmes n'étaient pas commutables, mais profondément répétitifs, invariables, intraduisibles d'un système graphique à l'autre.

11 Nous ne pouvons montrer ici comment la vocalisation progressive de ces marques entraîna une déterritorialisation, une universalisation du sens, ni comment se libéra ou comment fut produit un espace - qui cessant d'être pour l'écriture devint pour la vue. Une vue qui n'était plus celle de la lecture. Paradoxalement pour nous qui renvoyons systématiquement les "sauvages» du côté de l'oralité (Debray), leur expérience était celle d'un corps - la Terre - totalement recouvert de lettres (de graphèmes, d'entailles, de dessins).

12 Les «sauvages" n'avaient donc pas d'horizon, le monde (la Terre, les corps, les calebasses) ne leur étant pas donné à voir, mais à lire, selon une expérience toujours 
renouvelée de parcours de lecture, accessoirement d'écriture, parce que les étoiles sont toujours déjà-là, dans le ciel, comme un livre ouvert. C'est ce premier texte - le ciel qui a fait le premier lecteur, et non un scripteur s'autorisant de lui-même à s'inventer par l'écriture, sur la paroi rocheuse d'une grotte.

13 Parcourir les plages du Débarquement, en se donnant la perspective de l'assaillant marin, pour lequel la ligne d'horizon est une ligne de crêtes, plus ou moins fortifiée, ou en défenseur du mur de l'Atlantique embusqué dans sa casemate de commandement de tir comme à Longues-sur-Mer, protégé par une simple dalle de béton reposant sur quatre élégants poteaux d'acier, c'est faire malgré les apparences l'expérience de la réversibilité des points de vue.

Si les points de vue sont tels, c'est que les ennemis ont en commun la même définition de l'espace, le même géométral, lequel rend possible la comparaison de tous les points de vue, institue de fait un monde commun, une même objectivité, une même technorationalité, malgré un différend insurmontable (les nazis, les Alliés).

Paradoxalement donc, même si les lignes de l'assaut horizontal s'opposent, délimitant provisoirement l'espace de la bataille, c'est cette réalité de la limite visible de la Terre qui à la fois partage les ennemis et les conjoint dans la même appartenance à la sphère de la subjectivité.

Ils ne peuvent avoir la même ligne (même dans l'expérience limite du front où la ligne de crête sépare les deux tranchées), mais de ce qu'ils en ont chacun une, ils appartiennent au même monde de l'affrontement techno-scientifique, dont le substrat est ici la vue. Ce n'est donc pas l'affrontement qui rapprocherait, créant un site commun comme le ferait la vivante césure qui toujours génère de la différence (Hölderlin). L'espace moderne de l'affrontement supposant une chose publique est en son fond démocratique (et cela est bien évident dès la place publique des Cités-États italiens de la Renaissance et précisément de leurs représentations en peinture), même si, comme en Normandie se sont affrontés des démocraties parlementaires et un totalitarisme. D'ailleurs ces divergences socio-politiques fondamentales n'empêchèrent pas l'institution de musées de la bataille, qui ne purent, dès qu'ils eurent à en exposer les ruines, éviter de rendre les ennemis semblables, c'est-à-dire de même substance métaphysique. Le musée est d'essence démocratique.

L'idéal était dans le mémorial de Caen, du fait d'un film, de montrer la mobilisation totale de toutes les forces, de chaque côté de la Manche, mobilisation sans laquelle ces deux lignes d'horizon n'auraient pu être concrétisées comme limites du feu. La volonté n'est rien sans l'horizon de son exercice.

Que le musée d'Arromanches, situé au centre de l'ancienne zone de combat, attire plus un public mêlé d'anciens combattants des deux camps, suivi de l'inévitable arrièregarde des touristes de guerre, ou que le Mémorial fascine plus les vainqueurs et leurs descendants, venant y chercher une ligne d'horizon paradoxalement universelle et, pour ce faire, se transformant comme sens de l'histoire après la Shoah (un Mémorial de la Paix), les uns et les autres restent dans l'horizontalité commune dont l'irruption est co-extensive à celle des Temps modernes (Heidegger : Qu'est-ce qu'une chose?).

À la limite, le musée d'Arromanches deviendra avec le temps, comme le mémorial du champ de bataille de Douaumont, un musée pour la paix, tant il est vrai que les batailles contemporaines, qui voient s'affronter des masses guerrières dans l'anonymat le plus total, sont plutôt des lieux d'effondrement de toutes les valeurs pour lesquelles les 
combattants sont censés se battre. Les héros n'y peuvent être qu'inconnus, les soldats définitivement ébranlés, comme l'écrivait Patocka à propos de l'« expérience » du front de la Grande Guerre, ne survivant que dans un élan fraternel de suspension, par delà la ruine des valeurs du droit universel ou du sang ou du sol. La bataille contemporaine n'est plus le lieu d'une expérience (et cela probablement depuis Stendhal), sinon de l'effondrement de l'expérience subjective, dans laquelle s'évanouit toute ligne d'horizon.

Un héros ne peut plus y inscrire sa trace (cela devient même la chose la plus dangereuse qui soit de laisser des traces pour le satellite d'observation, le drone, une odeur pour un chien de combat, un écho pour le radar nocturne, une luminescence ou un spectre de chaleur pour le capteur infrarouge).

Brecht conseillait aux habitants du "pays sans prolétaire", dans les années trente:

"Ne laisse pas de traces ", réclamant ainsi la nécessaire rupture avec l'ancienne surface d'inscription - la moderne - en fait trop surencombrée, obsessionnellement saturée (l'extraordinaire extension du champ patrimonial).

Le héros inconnu, délesté de son ancienne destination épique: devenir une figure historique de la mobilisation de masse, afin de lui donner forme (la masse étant semblable à une quasi-matière), ce héros n'a plus d'horizon, mais en un tout autre sens que projectif, une ligne de fuite (Deleuze), ou mieux une ligne d'aire (Deligny).

Nos modernes touristes de guerre sont donc des nostalgiques de la ligne d'horizon, alors que l'expérience du combattant fut plutôt celle du devenir-animal, voire du devenir-minéral. Ils tentent de reconstituer une certaine normalité, celle qui se nourrit de la dialectique de deux horizons antagonistes, parce qu'ils espèrent un retour du sens, là précisément où il fit le plus défaut.

Mais une autre analyse serait possible, l'attente nostalgique en la résurrection du sens ou de la ligne d'horizon, pourrait être mêlée à une admiration pour un lieu (le champ de bataille) où ce sens et cet horizon ont été sacrifiés. Ce qui peut s'entendre à la suite de G. Bataille, comme expulsion et destruction qui ennoblissent le sacrifié, ou de Patocka encore, comme métanoïa, comme conversion philosophique en un fors le sens qui n'est pas celui du forcené de la psychiatrie, mais celui d'un passage à la limite quant au réel, au fait, au donné, vers ce qui leur donne sens.

Ces touristes rechercheraient le plus authentique (sans pouvoir le rejoindre) : la trace de ce qu'en ce lieu, là où les valeurs socio-politiques ont été suspendues, le nihilisme actif a triomphé sur toute œuvre, donnant lieu à une communauté inavouable, communauté sans communauté ou communauté négative, que J. L. Nancy définit par la différence ou le partage des voix. Communauté qui n'œuvre pas, constituée de singularités qui s'entr'aperçoivent ou plutôt s'entre-voisent, dans un espace qui n'existait pas avant elles, mais qu'elles contribuent à définir, sans horizon parce que scandé de mille manières.

Il est évident qu'aucune muséographie n'est actuellement capable de procurer un tel déplacement. Au contraire, la muséographie assure, par le biais de ses dispositifs pédagogiques que le sens n'a pas été ébranlé. Et comme au Mémorial de Péronne, que l'expérience du front était bien de même nature que celle des guerres antérieures, puisque la correspondance privée des soldats conservait le même contenu avant et après l'assaut. Si l'on postule ainsi de pseudo-continuités, c'est que l'histoire pour le musée est toujours rêveuse. 
Les musées de bataille de masse sont donc des institutions où le touriste vient s'approprier de la trace, alors que le combattant, s'il n'était pas un novice, devait tout faire pour s'évanouir et disparaître, sans laisser de traces.

Ne pas laisser de traces doit s'entendre encore d'une autre manière. Ne pas croire pouvoir en laisser, parce qu'elles seraient fallacieuses, inauthentiques. Ici, un malentendu doit être levé. Celui portant sur la question de la vérité de la bataille, sur l'authenticité de l'expérience et de sa relation. Comme pour tout événement contemporain, la réponse n'admet pas un pirandellien «à chacun sa vérité ", qui reste très perspectiviste (même au sens de Nietzsche).

Non pas que les points de vue des uns et des autres ne puissent seulement pas être comparés, mais que si certains pouvaient encore avoir un point de vue - ou la fiction d'un point de vue de surplomb pour l'état-major le mieux informé - d'autres n'étaient plus du tout dans la possibilité d'en avoir un. Ici, il faut rompre avec toute la problématique du point de vue, de l'horizon, du projet, de la perspective, du monde commun comme géométral où tous les points de vue sont en principe comparables. Là, il faut sortir de la modernité, de la représentation.

30 La différence n'est donc pas entre un point de vue borné de soldat, sous-informé parce qu'étroitement territorialisé, et une vue panoramique, de surplomb, fictivement totalement informée des éléments de la bataille. Car en fait dans l'action, pour le soldat, les éclats d'événements guerriers anéantissaient à chaque fois toute velléité de saisir une matière à penser, toute la surface psychique - la conscience subissant le feu devait être constamment restaurée contre les assauts de l'extérieur, devenant par làmême, suivant la leçon de Freud, un pare-excitations, absolument vital.

31 Le combattant fut dans la situation de l'homme moderne selon Baudelaire : ne pouvant plus faire de récit de ce qu'il a vécu parce qu'il n'a pas pu transformer ce qui lui est arrivé en traces qu'on peut intérioriser, et, à partir de là, se remémorer. Bref, en un mot : penser.

2 La vérité de l'événement n'est évidemment pas non plus dans les communiqués d'étatmajor, ou dans une historiographie qui ne connaît que ces archives. Mais alors, plus probablement, dans une littérature nocturne dont le point de départ est plutôt le constat de l'impossibilité de témoigner d'un vécu immédiat, malgré ou à cause pourtant de l'apparente multiplicité des témoignages. Ne peut-on ainsi considérer la multitude des témoignages diurnes, écrits sur Verdun, dans la décennie qui suivit, comme autant de tentatives personnelles de reconstructions psychiques?

3 Il faut donc faire son deuil de l'expérience vécue, expérience qui conduirait plutôt à l'extrême pauvreté du témoignage, voire au silence. Seul le silence authentifie le désastre du vécu. A contrario, seule la trace (littéraire, picturale ou cinématographique) fait être en suppléant à l'absence du vécu. Ce rapport à la trace n'est évidemment pas interdit à un public, s'il consent à ne pas s'approprier ce qui deviendrait un objet de consommation, mais plutôt à y habiter, comme le fait le véritable collectionneur, d'après W. Benjamin, qui pénètre les choses acquises pour en saisir l'énigme.

Une règle pour cette littérature, que l'on trouve chez $\mathrm{G}$. Duhamel à propos de Verdun : «Pour tout ce qui touche à Verdun de l'année 16, non, non, il n'y a pas de poésie, pas d'oubli, pas d'indulgence transfigurative de l'enfer ». 
$\mathrm{Du}$ : «ne pas laisser de traces», en passant par le «seule l'écriture institue authentiquement la trace de l'événement », un faisceau de thèmes se constitue où le « il y a des événements sans trace ", constitue le brin le plus énigmatique.

Si la ligne d'horizon est la condition pour qu'un sujet puisse être pointé, subjectivisant par là tout ce qui sera encadré par l'appareil perspectif, transformant la chose en objet, l'autre en ennemi qui est toujours un autre moi-même. Dès lors, la guerre moderne lui reconnaît un statut juridique (Convention de Genève).

artir du moment où l'on retire tous ses droits à cet autre, où on le dénationalise en instituant, comme le firent les nazis, un statut de citoyen de seconde zone, jusqu'à ne plus lui laisser - tout à fait provisoirement - que sa vie biologique et sa force de travail, alors il ne peut être considéré comme un ennemi auquel on ferait la guerre. Pour cette politique sauvage menée au cœur de la modernité (Lyotard), l'horizon n'est plus la limite idéale d'un projet. L'empire nazi de l'enracinement dans le sol et le sang, strictement continental, quasi incapable de se battre sur mer, n'avait que faire de l'horizon.

38 Les Juifs et les Tsiganes n'étaient pas des ennemis au sens strict pour les nazis, mais des poux. Radicalement désubjectivisés et évidemment pas dialectisés (comme chez Hegel entre le maitre et l'esclave, ou chez Marx, entre les classes). Ce qui conduit à remettre en cause la thèse selon laquelle le nazisme serait sous la dépendance de la métaphysique moderne du sujet, voire son achèvement (J. L. Nancy, Ph. LacoueLabarthe). Cette intrusion du sauvage dans le moderne serait plutôt la marque d'un changement d'époque pour la philosophe comme pour l'anthropologie. Le post-moderne, si l'on tient à ce terme.

L'enjeu concernerait toujours la trace : ne pas en laisser derrière soi, ne pas laisser de trace du crime, exterminer en faisant disparaitre systématiquement, ruiner les conditions communes de l'expérience, rendre incroyable l'événement contemporain. Tous points qui laissent penser que la véritable question est celle de l'émergence d'une nouvelle époque de la surface d'inscription dont le support ne serait plus la terre ou le corps, ni la vitre que l'on peut pénétrer d'un regard, selon l'étymologie aléatoire du mot perspective "raison pénétrante ». Une nouvelle ère dont le crime de masse serait l'acte de naissance, c'est-à-dire par l'extermination programmée, une politique et une esthétique de la disparition.

\section{RÉSUMÉS}

La question de l'intersubjectivité est toujours partie d'un dilemme : si ego est le foyer irréductible du sens, comment concevoir autrui, l'alter ego ? Comment déduire une multiplicité de foyers d'un foyer originaire? Pour une philosophie des appareils, la réponse est simple: l'appareil perspectif rend structurellement comparable tous les ego puisque pour le géométral tous les points de vue sont équivalents. À partir de son époque, l'objet et le sujet sont réversibles : ne parle-t-on pas du sujet d'un tableau ou d'un roman comme de son objet ? Dès lors, ce qui sépare deux armées ennemies modernes, la ligne de front, peut être conçue comme l'horizon d'une 
construction perspective, condition préalable de la position du point de fuite = point du sujet depuis Viator et Desargues. Les ennemis ont un sol commun qui rend possible une nouvelle définition du droit de la guerre (Convention de Genève). Mais pendant la seconde guerre mondiale, cette politique sauvage (Lyotard) a fait irruption au creux de la modernité : les Juifs et les Tziganes n'étaient pas pour les nazis des sujets/objets, mais des poux qu'il fallait faire disparaître. L'humanité est entrée dans l'époque de la disparition (Crime contre l'humanité).

\section{INDEX}

Mots-clés : appareil perspectif, bio-politique, crimes contre l'humanité, crimes de guerre, géométral, ligne d'horizon, musée d'histoire 